\title{
Discussion on the New Battery System of Energy Saving and Environmental Protection Materials
}

\author{
Li Junming ${ }^{1}$, Su Hainan ${ }^{1}$, Cao Shibing ${ }^{2}$, Yu Xin ${ }^{1}$, Yang Sen ${ }^{1}$, Xie Hongxu ${ }^{2}$, Fu Hai ${ }^{2}$, and Si \\ Hongxiao $^{1}$ \\ ${ }^{1}$ Dandong Power Supply Company, Liaoning Electric Power Company Limited, State Grid, China \\ ${ }^{2}$ Yantai Haibo Electrical Equipment Co. Ltd
}

Keywords: energy saving, environmental protection materials, online monitoring and management system, iron phosphate lithium battery

\begin{abstract}
Battery is an important part of the DC power supply system in the power supply system. As a DC power supply, it is mainly responsible for providing safe, stable and reliable power guarantee for the secondary system load in the power system to ensure the normal operation of relay protection and communication equipment. Therefore, the stability of the battery and the actual capacity that can be provided to the load in the discharge process are very important to ensure the safe operation of the power equipment.
\end{abstract}

\section{Introduction}

At present, the fixed valve-controlled sealed lead acid battery is commonly used in substation. This kind of battery design life is generally as long as 10 12 years (some manufacturers claim floating charging life of 15 years), the advantages of good sealing, cost-effective, do not need to supplement electrolyte and distilled water, no pollution, large current discharge capacity. However, the disadvantage of lead-acid battery is that the operation maintenance, operation environment requirements are high, if the environment is relatively bad, the operation and maintenance is not timely or not in place, the valve-controlled lead-acid battery will often appear in advance failure. If the bad environment occurs frequently and the inspection and maintenance work is not timely followed up, the lead acid battery failure years will be greatly advanced. With the popularization and application of new technologies such as low carbon economy technology and clean energy technology, new batteries of energy saving and environmental protection materials are used in operation and maintenance of DC power supply system in substation. As a new type of secondary power supply, with its features of long life, safe use, green environmental protection, high working voltage, large energy density, small self-discharge rate, no memory effect, small volume, etc., it has become a potential energy storage battery for the development of secondary battery industry, and is the future development direction of energy storage battery.

\section{The Cause of Valve - Controlled Lead - Acid Battery Failure}

\subsection{Operation and Maintenance of Valve-Controlled Sealed Lead Acid Battery}

Proper operation and maintenance of a valve-sealed lead acid battery is essential. Due to the short service time and little experience of valve-sealed lead-acid batteries in power plants, operation and maintenance personnel generally feel that the performance of valve-sealed lead-acid batteries is not enough to understand, and there is no chapter to follow. Or misinterpret "maintenance-free" as no maintenance at all. In fact, this only refers to the operation without adding acid and water, not without maintenance. The damage to the valve-controlled sealed lead-acid battery is caused by the operation and maintenance of the ordinary lead-acid battery for many years.

\subsection{Performance and Quality Problems of Valve-Controlled Sealed Lead Acid Batteries}

Early battery companies, due to the design and production process, there are uneven capacity, 
leakage, battery heat and loss of water, early failure and other problems, so that the battery reliability is reduced, the actual life is less than the expected years. Valve - controlled sealed lead acid battery, large internal resistance, to small current is not sensitive, small current can not be stored. For example, wind power is not suitable, there is memory, the amount of the first charge determines the future capacity of the battery. As the temperature of lead-acid battery decreases, the electrolyte is not easily diffused, and the chemical reaction of the active substances at the two poles is slow, which leads to the shortened power consumption time. This is why the battery is not durable in winter. At present, as backup power supply of substation, the battery group is basically composed of multiple lead-acid batteries in series. Lead-acid battery in the process of operation occasionally occur failures, the most serious case is the battery open circuit, followed by the battery capacity is insufficient, open circuit will cause the circuit breaker can not normally jump and close, and then burned transformer or high voltage room; However, the insufficient capacity will cause the DC load in the substation to drop to a very low voltage in a very short period of time, and then lead to the loss of power relay protection device, automatic device, circuit breaker trip mechanism, emergency lighting, etc., can not work.

\subsection{Problems in The Development of Valve-Controlled Sealed Lead-Acid Battery Industry}

At present, the illegal lead-acid battery and recycled lead production enterprises still exist, the technical equipment level is not high, the operation of standardized enterprises in the market competition is still at a disadvantage, affecting the healthy development of the industry. A few enterprises, in disregard of the national hazardous waste management requirements, illegally engaged in waste lead battery collection, storage and disposal activities, a large number of waste lead acid battery into the illegal recycling channels, making the standard recycling enterprises lack of competitive advantage in price. Lead-acid battery industry lack of technical support, economic and reasonable pollution control technology, especially the battery clean production technology industrialization operation is obviously insufficient, advanced technology promotion is not enough, restricted the technical progress of the industry.

\section{Research Significance of New Battery Based on Energy Saving and Environmental Protection Materials}

DC system in transformer substation, battery and charger in parallel, to relay protection and automatic device, automation equipment, circuit breaker jump switching mechanism such as important for power supply, DC load when the ac loss of electricity, the motor can output DC, batteries as the only DC power to power supply DC load. The failure of the accumulator in the case of emergency may lead to a major operation accident of the substation. Therefore, the battery is the core of the DC power system, and its performance quality affects the safe and stable operation of the whole substation. Due to the increasing degree of substation automation and intelligence and the popularization of unmanned duty, the DC power supply of substation plays an increasingly important role. Energy conservation and environmental protection new research direction developed battery raw material, the technology application in substation DC power system operations, as a new type of secondary power supply, with its long life, the use of safe, green environmental protection, high voltage, large energy density, small self-discharge, no memory effect, small size, etc., consciousness become secondary battery industry potential energy storage battery.

\section{Research on New Battery System of Energy Saving and Environmental Protection Materials}

With the spread and application of new technologies such as low-carbon economy technology and clean energy technology, the increase in GDP will not be fully proportional to carbon emissions, and at the same time provide a strong support for further achieving China's emission reduction goals. The lithium battery industry of energy-saving and environmental protection materials is in line with the guidance of national industrial policies. At present, battery industry is 
placed in an important position in national development strategies by all countries, and support from supporting funds and various policies is also very strong. China is also very supportive in this regard. The energy density of energy saving and environmental friendly lithium battery is high, which reduces the space of battery installation. Long service life, reduce the cost of battery replacement; Excellent temperature characteristics, saving the operating cost of the battery; Good safety performance, reduce the pollution of the environment; Superior charge-discharge performance guarantees the battery's service life; No memory effect, really maintenance free; Have intelligent management system. As the backup energy storage battery of communication power supply, DC power supply, UPS, EPS and other equipment, the lithium battery pack of energy-saving and environmental protection materials adopts iron phosphate lithium battery cell to form a group, and the battery pack is protected and managed by charge and discharge through the battery management system (BMS). Through the series - and management of different Numbers of lithium iron phosphate cells, it can flexibly complete a variety of large capacity lithium battery packs such as $10 \mathrm{ah}-400 \mathrm{ah}$, and the voltage of lithium battery pack can be flexibly combined from $24 \mathrm{~V}$ to $600 \mathrm{~V}$.

\subsection{System Introduction}

The CAN communication mode used in the traditional lithium battery management system is changed to RS485 communication, which is more suitable for the power system and industrial and mining enterprises in the substation DC system, UPS, EPS and other backup power fields. The real-time control of program-controlled DC power supply is realized, and the transient response ability and real-time performance of program-controlled DC power supply are effectively improved due to the powerful sequential circuit processing capacity and digital signal processing capacity of the microprocessor. Adopting temperature drift compensation circuit technology, the precision of the circuit in the wide temperature range can meet the design requirements. Digital PID control is adopted to directly convert the digital error control signal into the output control signal through the DAC circuit, which provides the output control signal to the isolated drive circuit to realize the real-time control of the program-controlled DC power supply. TCP/IP protocol stack lwip technology is used to realize the rapid communication between PC and lithium battery BMS management system. The PC software communicates with multiple sets of BMS through the Intranet, and transmits data such as battery voltage, current, residual capacity SOC, health status $\mathrm{SOH}$, system status and so on to the PC software for display and storage. Data transmission through the cloud service technology, support android, IOS SDK interface access technology, mobile APP can remotely view the lithium battery data and charts. Lithium battery adopts the mode of minimum module cold connection, which not only avoids the problem of group replacement due to the single cell problem, but also reduces the replacement cost and the replacement time. This is shown in Figure 1.

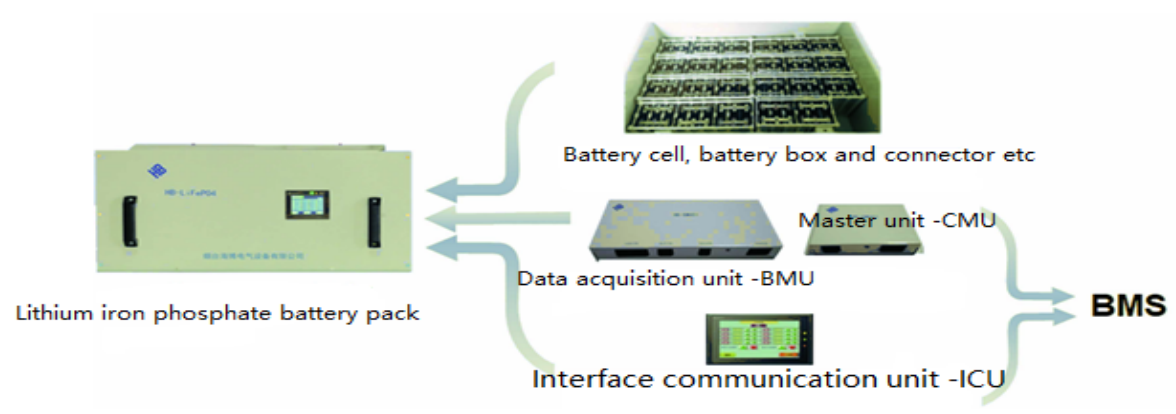

Figure 1 System structure diagram

\subsection{Working Principle}

Smart battery management system is the guarantee of the safe operation of the battery pack, not 
only require high precision, but also has all kinds of protection and alarm functions, various communication interfaces have commonly used protocols of power substation automation system, but also can realize industrial field centralized management of the multiple sets of lithium battery back-up power system, efficient management, history data graphical, cloud services, remote maintenance, etc. The "data acquisition unit" of the management system is responsible for collecting the voltage, charge and discharge current, temperature and other data of each cell in the cabinet, and for the balanced management and charge and discharge protection of the cell. The "main control unit" collects the data of the "data acquisition unit", conducts centralized analysis and processing of the battery pack data, and carries out alarm and control according to the battery pack operation status. At the same time, "main control unit" also completes the battery working current measurement, charge and discharge control. This is shown in Figure 2.

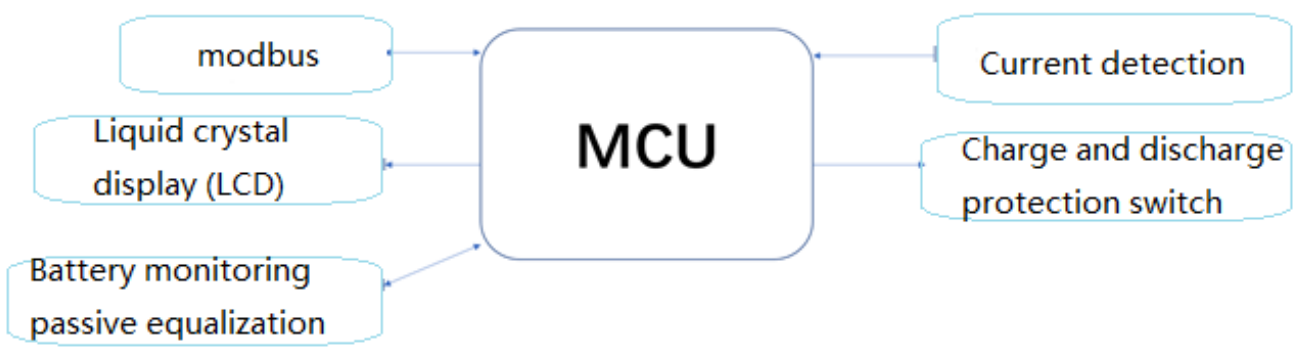

Figure 2 BMS functional block diagram

Master control unit CMU, complete SOC estimation, current monitoring, processing information and alarm functions. The data acquisition unit BMU, each data acquisition unit can complete the data acquisition, voltage balance and charge and discharge protection functions of 8 to 24 sections of the cell, so as to maintain the consistency of the charging voltage of the parallel cell, so as to protect all the cells from being hurt during the charging process. At the same time, the data acquisition unit will collect the cell voltage, temperature and other information through the bus transmission to the main control unit. The interface communication unit ICU is the human-computer interaction interface of the battery management system, which CAN communicate through the RS485/CAN bus and display various operating parameters and failure conditions of the system. All models are designed to industry standards and are suitable for use in a variety of environments.

\subsection{Characteristics}

(1) Discharge rate: generally 2-5c, up to 10C

(2) Self-discharge rate: $<3 \%$

(3) Test energy density mAh/g: 110-165

(4) Number of cycles: 2000

(5) Operating temperature: $-40--60^{\circ} \mathrm{C}\left(-40^{\circ} \mathrm{C}\right.$ discharge capacity is greater than $90 \%, 55^{\circ} \mathrm{C}$ can discharge at full capacity does not affect the service life.)

(6) It is not affected by the temperature, so there is no need for temperature compensation.

(7) Maintenance-free: lithium iron phosphate battery pack does not need to regularly check the charge and discharge, is really maintenance-free

(8) Lithium iron phosphate battery has no memory effect and can be discharged fully under any circumstances

(9) It is equipped with battery management system (BMS) to complete individual battery detection, balance and other control, and all data can be uploaded, which is completely intelligent.

(10) The positive electrode material is lithium iron phosphate

\section{Conclusion}

The new battery system of energy saving and environmental protection materials has completely 
solved the potential safety problems of various batteries under the condition of strong impact, fall, acupuncture, open fire, overcharge and high temperature. In the whole substation battery life cycle calculation, compared with the traditional lead-acid battery, the battery using the new energy-saving environmental protection materials can save more than $40 \%$ of the investment, and the new energy-saving environmental protection material battery has no pollution to the environment, high recycling rate, social benefits are very significant.

\section{References}

[1] Ma Cunbing. Maintenance technology of lead-acid battery [N]. E-paper. In 2002.

[2] Ng Singfai, Eugene cross, Lin Zugeng. Research on carbon anode materials for lithium ion batteries [J]. Power technology. 01, 1998.

[3] Zhong Junhui. Research progress of carbon materials for anode pyrolysis of lithium ion batteries [J]. Power technology; 01, 1998.

[4] Hu Shaojie, Xu Baobo. Development and prospect of lithium ion battery industry [J]. The battery.04, 2000.

[5] Mai Liqiang, Zou Zhengguang, Chen hanyuan, Research progress of anode materials for lithium ion batteries [J], Material guide, 07.2000. 